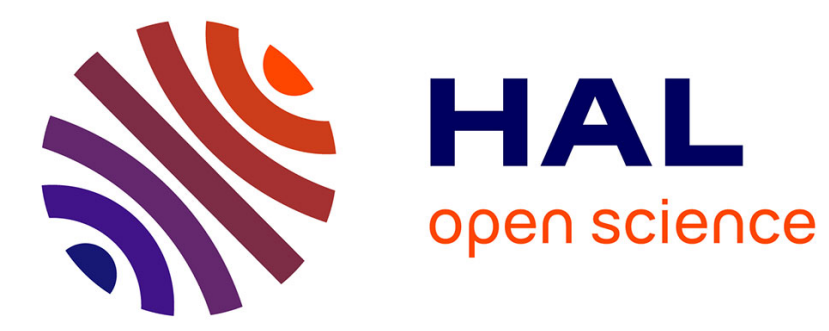

\title{
Efficient laser-overdense plasma coupling via surface plasma waves and steady magnetic field generation
}

A Bigongiari, M Raynaud, C Riconda, Anne Héron, A Macchi

\section{To cite this version:}

A Bigongiari, M Raynaud, C Riconda, Anne Héron, A Macchi. Efficient laser-overdense plasma coupling via surface plasma waves and steady magnetic field generation. Physics of Plasmas, 2011, $10.1063 / 1.3646520$. hal-01327425

\section{HAL Id: hal-01327425 \\ https://hal.science/hal-01327425}

Submitted on 6 Jun 2016

HAL is a multi-disciplinary open access archive for the deposit and dissemination of scientific research documents, whether they are published or not. The documents may come from teaching and research institutions in France or abroad, or from public or private research centers.
L'archive ouverte pluridisciplinaire $\mathbf{H A L}$, est destinée au dépôt et à la diffusion de documents scientifiques de niveau recherche, publiés ou non, émanant des établissements d'enseignement et de recherche français ou étrangers, des laboratoires publics ou privés. 


\title{
Efficient laser-overdense plasma coupling via surface plasma waves and steady magnetic field generation
}

\author{
A. Bigongiari, ${ }^{1,2}$ M. Raynaud, ${ }^{1}$ C. Riconda,${ }^{2}$ A. Héron, ${ }^{3}$ and A. Macchi ${ }^{4}$ \\ ${ }^{1}$ CEA/DSM/LSI, CNRS, Ecole Polytechnique, 91128 Palaiseau Cedex, France \\ ${ }^{2}$ TIPS/LULI, Université Paris 6, CNRS, CEA, Ecole Polytechnique, 3, rue Galilée 94200, Ivry-sur-Seine, \\ France \\ ${ }^{3}$ CPHT, CNRS, Ecole Polytechnique, 91128 Palaiseau Cedex, France \\ ${ }^{4}$ Consiglio Nazionale delle Ricerche, Istituto Nazionale di Ottica (CNR/INO), Dipartimento di Fisica "E. \\ Fermi," Largo B. Pontecorvo 3, 56127 Pisa, Italy
}

(Received 1 July 2011; accepted 9 September 2011; published online 11 October 2011)

\begin{abstract}
The efficiency of laser overdense plasma coupling via surface plasma wave excitation is investigated. Two-dimensional particle-in-cell simulations are performed over a wide range of laser pulse intensity from $10^{15}$ to $10^{20} \mathrm{~W} \mathrm{~cm}^{-2} \mu \mathrm{m}^{2}$ with electron density ranging from 25 to $100 n_{c}$ to describe the laser interaction with a grating target where a surface plasma wave excitation condition is fulfilled. The numerical studies confirm an efficient coupling with an enhancement of the laser absorption up to $75 \%$. The simulations also show the presence of a localized, quasi-static magnetic field at the plasma surface. Two interaction regimes are identified for low $\left(I \lambda^{2}<10^{17} \mathrm{~W} \mathrm{~cm}^{-2} \mu \mathrm{m}^{2}\right)$ and high $\left(I \lambda^{2}>10^{17} \mathrm{~W} \mathrm{~cm}^{-2} \mu \mathrm{m}^{2}\right)$ laser pulse intensities. At "relativistic" laser intensity, steady magnetic fields as high as $\sim 580 \mathrm{MG} \mu \mathrm{m} / \lambda_{0}$ at $7 \times 10^{19} \mathrm{~W} \mathrm{~cm}^{-2} \mu \mathrm{m}^{2}$ are obtained in the simulations. (C) 2011 American Institute of Physics. [doi:10.1063/1.3646520]
\end{abstract}

\section{INTRODUCTION}

The recent development of Ti-Sapphire lasers providing ultra-short $(\tau \leq 100 \mathrm{fs})$ intense $\left(I \lambda_{0}^{2} \geq 10^{17} \mathrm{~W} \mathrm{~cm}^{-2} \mu \mathrm{m}^{2}\right)$ IR laser pulse ${ }^{1}$ and the plasma mirror technique, ${ }^{2}$ which permits high pulse contrast $\left(>10^{11}\right)$, allows the creation of a sharp-edged overdense plasma before any eventual smoothing of the density gradient by hydrodynamic expansion in thick plasmas $\left(L_{x} \gg c / \omega_{p e}\right.$, where $L_{x}$ indicates the plasma thickness, $\omega_{p e}$ is the plasma frequency, and $c / \omega_{p e}$ is the skin depth). In such sharp-edged overdense plasmas, the electromagnetic energy is weakly absorbed in an optical skin depth by collisional processes and through collisionless mechanisms ${ }^{3}$ such as sheath inverse bremsstrahlung, ${ }^{4} \vec{J} \times \vec{B}$ heating, ${ }^{5}$ vacuum heating, ${ }^{6}$ and anomalous skin-layer heating. ${ }^{7}$ Owing to the ultra-short laser pulse duration and the steep density profile of the plasma, the laser reflection is very high and can easily exceed $80 \%$ (Ref. 8 ), thus limiting the production of high energetic particles. This point is a drastic limitation in all applications related to particle acceleration and for the fast ignitor scheme in the framework of the inertial confinement fusion. ${ }^{9,10}$

To overcome these difficulties, new mechanisms have been investigated in order to improve laser absorption and electron acceleration by considering structured targets ${ }^{11-16}$ which, in particular, allow the excitation of surface plasma waves (SPWs). ${ }^{17}$ These waves are supported by a stepwise profile, overdense $\left(\omega_{p e}>\omega_{0}\right.$, with $\omega_{0}$ the laser frequency) plasma when the condition for resonant excitation are satisfied. SPWs propagate along the plasma-vacuum interface and are characterized by a localized, high frequency, resonant electric field. In a previous work limited to a $10^{18} \mathrm{~W} \mathrm{~cm}^{-2} \mu \mathrm{m}^{2}$ laser intensity, ${ }^{13}$ we have shown the possibility of resonant excitation of the SPW by ultrashort (60 fs) high intensity laser pulse in overdense $\left(n_{e}=25 n_{c}\right.$, where $n_{c} \equiv \omega_{0}^{2} m_{e} / 4 \pi e^{2}$ is the cut-off density at which $\omega_{p e}=\omega_{0}$ ), pre-structured plasma. A dramatic increase of both the laser absorption (up to the 70\%) and the electron energy (several $\mathrm{MeV}$ ) was observed. A high local amplified electric field was also found, which has interesting consequences on energetic electron bunch creation. It is worth noticing that high contrast and ultrashort duration of the laser pulse are essential prerequisites for SPW absorption to work at very high intensities. The interaction time should be short enough to avoid the grating structure to be washed out by the plasma expansion, while too strong a prepulse would destroy the grating even before the short pulse interaction. This latter effect, which has been likely the main limiting factor for experimental investigations of SPW absorption in the past, may now be prevented by the above mentioned plasma mirror techniques for pulse cleaning, motivating further theoretical and numerical work.

In this paper, we present two-dimensional particle-in-cell (PIC) simulations where the plasma surface is initially preformed, so that the SPW excitation conditions are fulfilled. We wish here to investigate the transition from the nonrelativistic to the relativistic regime, observing the laser overdense plasma coupling via surface plasma wave over a wide range of pulse intensity (from $10^{15}$ to $10^{20} \mathrm{~W} \mathrm{~cm}^{-2} \mu \mathrm{m}^{2}$ ). The role of the electron density on the coupling efficiency will be also investigated increasing the electron density from 25 to $100 n_{c}$. It is found that even increasing the laser intensity or/and the electron density, the laser absorption when exciting SPW remains higher than $60 \%$. So the proposed setup may have important applications, for example, in electron and ion acceleration schemes, or creation of positron jets from intense laser solid interaction. ${ }^{18}$ This approach could also enable efficient generation of energetic electrons in a solid-density material reducing pre-heating, allowing studies 
of transport in warm dense matter ${ }^{19}$ and dependence on the crystalline structure. $^{20}$

We also show via our simulations that the SPW excitation enhances the generation of a quasi-static magnetic field near the plasma surface, possibly leading to higher field values with respect to other known mechanisms. ${ }^{21}$ These latter include nonparallel electron density gradient and temperature, $^{22}$ the radiation pressure associated with the laser itself, ${ }^{9}$ and/or the current of fast electrons generated during the interaction. ${ }^{23}$ The growth of intense magnetic fields localized in the vicinity of the interaction layer can play an important part as, for instance, it was found to determine the divergence of the beam of energetic particles entering the plasma $^{24,25}$ and the number of particle in the beam. ${ }^{26}$

Moreover, we remind that surface plasma wave can also appear on flat (non-structured) overdense plasmas using double step gradient density profile. ${ }^{27}$ In such case, however, the experimental laser SPW excitation seems harder to reach than our following scheme. Finally, it should be mentioned that SPW can also arise by decay process, ${ }^{28}$ thus the associated generation of a quasi-static magnetic field is relevant for many situations of interest. Thus, the SPW excitation will have the practical advantage of allowing the creation of $\mathrm{MeV}$ particles and hundred megagauss (MG) quasi-static magnetic generation, in not too high laser pulse intensity range $\left(10^{18-19} \mathrm{~W} \mathrm{~cm}^{-2} \mu \mathrm{m}^{2}\right)$. The paper is organized as follows. The code and the framework for our numerical studies are detailed in Sec. II. The laser absorption and the local surface electric field enhancement are first investigated as a function of the laser intensity in Sec. III. Two interaction regimes are found for low $\left(I \lambda_{0}^{2}<10^{18} \mathrm{~W} \mathrm{~cm}^{-2} \mu \mathrm{m}^{2}\right)$ and high $\left(I \lambda_{0}^{2} \gtrsim 10^{18} \mathrm{~W} \mathrm{~cm}^{-2} \mu \mathrm{m}^{2}\right)$ laser pulse intensities. In Sec. IV, we examine the damping of the SPW via energy transfer to hot particles. The role of the electron density is then investigated in Sec. V. In both low and high laser intensity regimes, we observe the presence of a very strong static magnetic field localized at the plasma surface, which can reach the value of $\sim 580 \mathrm{MG}$ for $I \lambda_{0}^{2}=7 \times 10^{19} \mathrm{~W} \mathrm{~cm}^{-2} \mu \mathrm{m}^{2}, \lambda_{0}=1 \mu \mathrm{m}$. These results are presented in Sec. VI.

\section{PIC SIMULATIONS}

The two-dimensional PIC simulations are performed by using the code EMI2D. ${ }^{29}$ An overdense plasma with $n_{e}=25-100 n_{c}$ is considered with a steep density gradient along the $x$ direction of the $(x, y)$ simulation plane. The plasma is initially described by a Maxwellian distribution with electron temperature $T_{e}=1 \mathrm{keV}$ (thermal velocity $v_{t h}=0.044 c$ ) and ion temperature $T_{i}=T_{e} / 10$ (here $m_{i} / m_{e}$ =1836, where $m_{i}$ and $m_{e}$ are the ion and electron mass, respectively). The charge of the ions was $Z=1$. The incoming laser beam is $P$-polarized (the only possible polarization for surface plasma wave excitation), so that the laser electric field is in the $(x, y)$ simulation plane. The laser has angular frequency $\omega_{0}$ and wavelength $\lambda_{0}$ and is characterized by a temporal Gaussian shape corresponding to a pulse duration of $60 \mathrm{fs}$ full width at half maximum. Different laser intensities have been studied starting at low intensities $\left(10^{15}-10^{17}\right) \mathrm{W} \mathrm{cm}^{-2} \mu \mathrm{m}^{2}$ up to $10^{20} \mathrm{~W} \mathrm{~cm}^{-2} \mu \mathrm{m}^{2}$ to investi- gate relativistic effects. The surface wave is resonantly excited providing the phase matching conditions $\omega_{0}=\omega$ and $k_{0 y}=k$, where the subscript $y$ indicates the $y$ component of the laser field wave vector $k_{0}=\omega_{0} / c$. As the phase velocity of a surface wave is lower than the light velocity to satisfy the phase matching conditions, we can consider a plasma surface having a periodic shape along the $y$ direction such that $k=k_{0}\left(\sin \theta+\lambda_{0} / a\right)$, where $a$ denotes the surface density period and $\theta$ is the laser incidence angle.

The dispersion relation of the surface plasma wave in the limit of low temperature $v_{t h} / c \ll 1$, as it is our case at the beginning of the interaction, takes the form $k^{2} c^{2} / \omega^{2}$ $=\left(\omega^{2}-\omega_{p e}^{2}\right) /\left(2 \omega^{2}-\omega_{p e}^{2}\right)$. The phase matching conditions combined with the surface wave dispersion relation lead to the resonance condition, in terms of plasma and laser parameters, $\sin (\theta)+\lambda_{0} / a=\sqrt{\left(1-n_{e} / n_{c}\right) /\left(2-n_{e} / n_{c}\right)} \simeq 1$, where the last equality is valid for $n_{e} / n_{c} \gg 1$. In the following, we have taken an incidence angle of $\theta=30^{\circ}$ with a modulation periodicity of $2 \lambda_{0}$. The modulation depth was equal to $\lambda_{0} / 2$.

In the simulations, the box size is $6 \lambda_{0}$ along the $y$ direction and $46 \lambda_{0}$ along the $x$ direction. The plasma occupies the right semispace for an extension of about $8 \lambda_{0}$, so that there is a vacuum length of $38 \lambda_{0}$ in front of the target which allows spatial expansion of particles, minimizing edge effects. The particle boundary conditions are periodic in the $y$ direction, and the fast electrons reaching the right box side are cooled in a buffer. The grid size used was $\Delta x \approx 5 \lambda_{d}$ and $\Delta y \approx 4 \lambda_{d}$ (thus depending on the initial plasma density taken in the simulation), where $\lambda_{d}=v_{t h} / \omega_{p}$ is the Debye length, in order to resolve correctly lengths below the plasma skin depth $\lambda_{p}=c / \omega_{p}$. The time interval used was $\Delta t \approx \Delta y / c$, and the number of particles per cell $\mathrm{NP}=100$. In the simulations, lengths and time are normalized, respectively, to the inverse of the laser wave vector $k_{0}^{-1}$ and the inverse of the laser frequency $\omega_{0}^{-1}$. The electric and magnetic fields are normalised to $E_{0}=B_{0}=m_{e} c \omega / e$. If $\lambda_{0}=1 \mu m, E_{0}=3 \times 10^{10} \mathrm{~V} \mathrm{~cm}^{-1}$ and $B_{0}=107 \mathrm{MG}$. In order to compare the effects of the SPW excitation with the non-resonant case, most of the simulations were complemented with simulation having the same characteristics but a flat plasma-vacuum interface.

\section{SURFACE PLASMA WAVE EXCITATION}

The surface plasma wave excitation is first studied for the case $n_{e}=25 n_{c}$. For the physical parameter considered in the simulation, the surface wave excited via laser-grating coupling is such that the $\mathrm{x}$-component of the electric field is larger by roughly a factor of $\left(n_{e} / n_{c}\right)^{1 / 2}$ than the y-component. ${ }^{13}$ We thus consider in Figure 1 as the reference field for the surface wave, the amplitude of the electric field along the $x$-direction, labeled $E_{s w}$, at $\omega t_{E \max }=460$, i.e., at the time when it attains the maximum value (the laser pulse peak impinges onto the modulated surface at $\omega t_{\max }=440$ ). For reference, the tips of the modulated surface are located at $x=240 k^{-1}$. The SPW field structure is localized at the modulated surface and has the expected value for the $k$ vector, that is, $k \sim k_{0}=2 k_{0, y}$ as visible in the figure, and verified by considering the spatial Fourier transform of the field. The oscillation frequency is $\omega$, in agreement with the resonant 


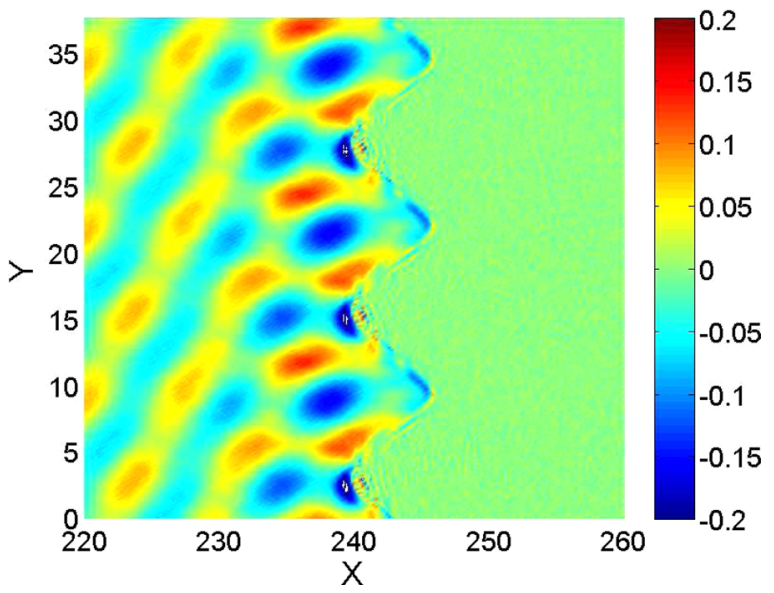

FIG. 1. (Color online) $x$ component of the SPW field and the exciting laser field in the $x y$ plane (left: vacuum, right: plasma, the modulated surface being at $x=240)$, at its maximum amplitude $\left(\omega t_{E \max }=460\right)$. The SPW is excited at the plasma surface by a pulse of intensity $I \lambda_{0}^{2}=10^{16} \mathrm{~W} \mathrm{~cm}^{-2} \mu \mathrm{m}^{2}$. The field is in units of $E_{0}$, and the lengths are in units of $k^{-1}$.

excitation condition of the SPW. The same features of the field are observed on the plasma surface varying the laser intensity from $\left(10^{15}-10^{20}\right) \mathrm{W} \mathrm{cm}^{-2} \mu \mathrm{m}^{2}$, only the value in time for which a maximum of the field is observed $\omega t_{E \max }$, which varies in time ranging between 450-520.

The high efficiency of the SPW laser coupling can be estimated via the laser absorption at the end of the simulation $\left(\omega t_{\text {end }}=1000\right)$ reported in the Fig. 2 as a function of the laser parameter $a_{0}=E_{L} / E_{0}=e E_{L} / m_{e} c \omega$. The case where the SPW is excited is in dark gray in contrast with the case where no SPW is present (case of a flat plasma surface) in light gray at different laser intensity. In the simulations where the surface wave is present, we observe enhanced laser absorption up to $77 \%$ for $I \lambda_{0}^{2}>10^{19} \mathrm{~W} \mathrm{~cm}^{-2} \mu \mathrm{m}^{2}$ while it is just $40 \%$ for the case of a flat surface. We observe that the absorption change abruptly its slope at $I \lambda_{0}^{2}>10^{17} \mathrm{~W} \mathrm{~cm}^{-2} \mu \mathrm{m}^{2}$, for both cases.

We first discuss briefly the results of the case of a flat surface. According to previous work, ${ }^{30,31}$ the drop of absorption with increasing the laser intensity from $a_{0} \ll 1$ to $a_{0}=1$

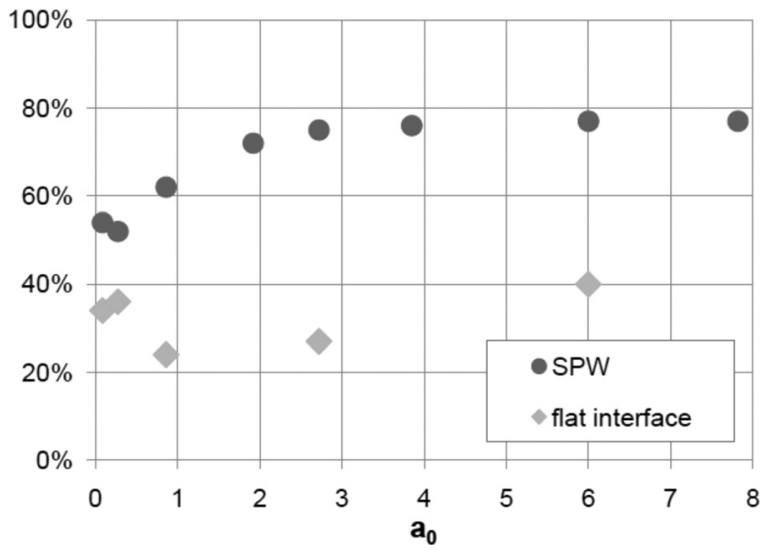

FIG. 2. Plot of the laser absorption ratio as a function of the laser parameter $a_{0}=e E_{L} / m_{e} c \omega=0.86 \sqrt{I \lambda_{0}^{2} /\left(10^{18} \mathrm{~W} \mathrm{~cm}^{-2} \mu \mathrm{m}^{2}\right)}$. In dark grey filled circle, the laser absorption ratio in the case where the surface plasma wave is excited, and in light grey filled diamond, the laser absorption ratio in the case of a flat interface. seen in Figure 2 can be interpreted by the fact that the ponderomotive pressure of the laser on the plasma locally steepens the electron density. It induces a decrease of the absorption due to mechanisms involving the presence of a density gradient. This is consistent with Refs. 30 and 31 where it was found that, for a given initial value of $L_{n} / \lambda_{0}$, where $L_{n}^{-1}=(1 / n) d n / d x$ is the typical scale of density variation, the absorption would decrease with increasing intensity, and for a given intensity, the absorption would decrease as $L_{n} / \lambda_{0}$ becomes smaller. At higher intensities, as $a_{0}$ increases from 1 to 6 , the absorption increases and reaches $40 \%$. This is not surprising since the dominant absorption mechanisms, vacuum heating and $\vec{J} \times \vec{B}$ heating ${ }^{3}$ become more efficient. It is to be noticed that there are many simulations in the literature in regimes similar to the one we investigate here. ${ }^{31,32}$ For example, one of the most recent work on ultra intense, ultrashort laser pulse (12 fs) interaction based on $1 D$ PIC simulations ${ }^{33}$ gives absorption values of the order of our flat surface result. However, to our knowledge, no systematic scan in intensity has been performed for laser absorption in thick overdense targets in the ultra high intensity regime as done here.

The laser absorption in the case of resonant excitation of the SPW can be related to the SPW growth and to the SPW damping by the electrons. The absorption curve of the laser can be directly linked to a field amplification factor defined as $\eta=E_{s w} / E_{L x}$, where $E_{S w}$ is the component of the SPW field along the $x$-direction and $E_{L x}$ is the laser one. This $\eta$ factor is calculated extracting the maximum $E_{s w}$ field values from the simulation at $\omega t_{E \max }$ (this time slightly varying with the laser intensity). As shown in Fig. 3, where $\eta$ is plotted as a function of the laser parameter $a_{0}$, the value of the amplification factor depends on the laser pulse field and ranges from a maximum value of 7.3 for $I \lambda_{0}^{2}=10^{15} \mathrm{~W} \mathrm{~cm}^{-2} \mu \mathrm{m}^{2}$ to a minimum value of 3.2 for $I \lambda_{0}^{2}=10^{18} \mathrm{~W} \mathrm{~cm}^{-2} \mu \mathrm{m}^{2}$. The parameter $\eta$ then rises again for increasing intensity above the relativistic limit, reaching a saturation value of $\sim 5$ at $I \lambda_{0}^{2}=7 \times 10^{19} \mathrm{~W} \mathrm{~cm}^{-2} \mu \mathrm{m}^{2}$.

In general, the saturation value of $\eta$ should depend on the rate at which the SPW transfers its energy to particles, via Landau damping or particle acceleration, or to fields,

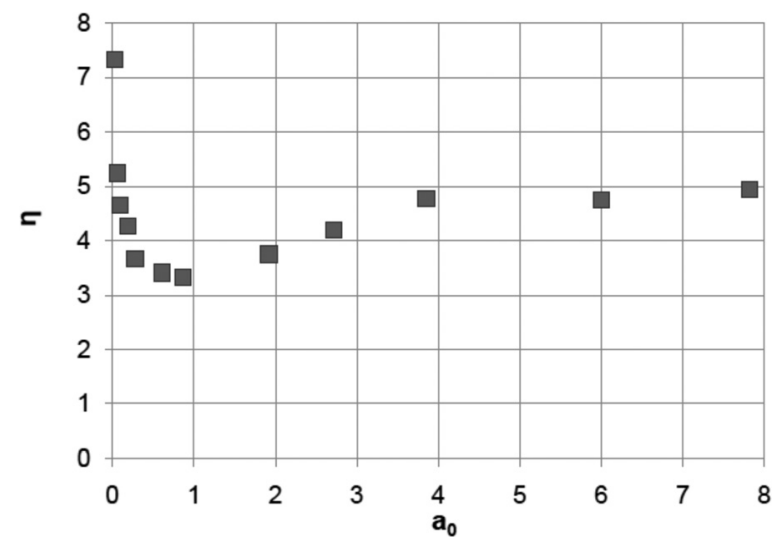

FIG. 3. Plot of the amplification factor $\eta=E_{s w} / E_{L x}$ as a function of the laser parameter $a_{0}$, where $E_{s w}$ is the component of the SPW field along the $x$-direction and $E_{L x}$ is the laser one. 
e.g., by re-emitting electromagnetic radiation. If the effective damping rate resulting from such processes is high enough, a steady state in which $\eta$ remains constant is reached during the laser pulse. From Figures 2 and 3, we can infer that, at the transition from the non-relativistic to the relativistic limit $\left(a_{0} \sim 1\right)$, the typical damping time becomes comparable to the SPW growth time; the wave does not grow further as its energy is immediately transferred to particles or fields. For this reason when $a_{0} \sim 1$, the surface wave shows a minimal amplification while the laser absorption increases significantly with respect to the case at lower intensity.

In the lower intensity cases, $\eta$ is larger, presumably because the wave is damped more slowly by the electrons. This is confirmed by observing that (1) the maximum value of $\eta$ is reached later in time than in the cases where $a_{0} \gtrsim 1$ and (2) after the laser is over, the SPW and the related electron heating last much longer than for higher intensities, for which the SPW wave quickly vanishes after the laser pulse.

We emphasize that the field amplification and absorption enhancement related to the SPW excitation are still very efficient in the relativistic regime, i.e., in the intensity range $10^{18}-7 \times 10^{19} \mathrm{~W} \mathrm{~cm}^{-2} \mu \mathrm{m}^{2}$. For instance, absorption in this range varies from $72 \%$ at $I \lambda_{0}^{2}=10^{18} \mathrm{~W} \mathrm{~cm}^{-2} \mu \mathrm{m}^{2}$ to $75 \%$ at $I \lambda_{0}^{2}=7 \times 10^{19} \mathrm{~W} \mathrm{~cm}^{-2} \mu \mathrm{m}^{2}$. This also suggests that relativistic detuning which will tend to decrease the resonant frequency of the surface wave ${ }^{12}$ does not affect the laser-SPW coupling significantly; possibly, it may be compensated by local electron heating ${ }^{17}$ that instead will tend to increase the SPW resonant frequency.

\section{DAMPING OF THE SPW}

Collisionless absorption and generation of suprathermal electrons in high intensity laser interaction with solid targets have been described in various ways..$^{5,6,30,34}$ The common feature to those different mechanisms is the combination of a sharp density gradient and of an oscillating force component perpendicular to the surface. For example, Brunel's "not-so resonant absorption" requires oblique incidence and $P$-polarization. The surface wave excited via laser-grating coupling has a perpendicular field component that can be quite larger than the corresponding component of the laser field (see Sec. III), hence leading to higher absorption and electron energies and resulting in an effective damping for the surface wave. In the presence of the surface wave, two additional effects might also influence the generation of suprathermal electrons. First, the surface wave field is evanescent on the vacuum side, so that the associated steady ponderomotive force is directed towards the incoming laser and may push electrons in such direction. A small fraction of electrons ejected in vacuum is observed indeed; however, this effect seems not to play an important role in our simulations. Second, the surface wave has a longitudinal field component along the propagation direction $(y)$ that may accelerate along the surface electrons whose velocity is close to the phase velocity of the surface wave. However, in the regime of our simulations $\left(\omega_{p} \gg \omega\right)$, we find that the longitudinal field component is small (see also Ref. 13) and only relativistic electrons may get in phase with the wave. Actually, electrons must ac- quire a velocity also in the $y$ direction for reasons of conservation of light wave momentum, since for oblique incidence and non-vanishing energy, absorption part of the parallel $(y)$ component of the laser wave momentum must be also absorbed by the plasma. Such absorption of momentum is mediated by nonlinear forces.

To explain the different behaviour in the two regimes, high and low intensities, we recall that the scale of variation of the SPW field inside the plasma is $L_{E, p l}$ $\sim k_{0}^{-1}\left(n_{e} / n_{c}-2\right)^{1 / 2} \sim c / \omega_{p e}$ (in the high density, low temperature limit). In the "vacuum" side, we have $L_{E, v}=\lambda_{0}$. Thus, the typical scales in the direction perpendicular to the surface where the electron can feel the effect of the SPW are either the electron skin depth or the evanescence length in vacuum, i.e., the laser wavelength. Let define this excursion length as $\Lambda_{o s c} \equiv v_{o s c, s w} / \omega$ where $v_{o s c, s w}=p_{o s c, s w} / m \gamma_{o s c, s w}$ is the electron oscillation velocity in the SPW field with $\gamma_{o s c, s w}=\left(1+p_{o s c, s w}{ }^{2} / m^{2} c^{2}\right)^{1 / 2}$ and $p_{o s c, s w}=e E_{s w} / \omega$. For low laser intensity $I \lambda_{0}^{2} \sim 10^{15} \mathrm{~W} \mathrm{~cm}^{-2} \mu \mathrm{m}^{2}$ and $n_{e} / n_{c}=25$, we have $\Lambda_{o s c} \lesssim L_{E, p l}$ and $\Lambda_{o s c}<L_{E, v}$ both on the vacuum and the plasma sides, thus electrons may gain energy only via the ponderomotive force. The resulting typical time scale for damping is such that the wave can still grow to large values.

For relativistic values such that $I \lambda_{0}^{2} \gtrsim 10^{18} \mathrm{~W} \mathrm{~cm}^{-2} \mu \mathrm{m}^{2}$, $\Lambda_{\text {osc }}$ increases up to its maximum value $\sim 0.16 \lambda_{0}$ and, thus, becomes comparable to or larger than $L_{E, v}$ and $L_{E, p l}$. In this regime, vacuum heating enhanced by the presence of the field of the surface wave sets in "enhanced vacuum heating" mechanism. Analysis of phase space shows that the maximum momentum normal to the surface, and acquired by an electron a few cycles after the maximum of the laser pulse has reached the plasma, is given by $p_{\mathrm{MAX}, x} \simeq 2.8 p_{o s c, s w, x}$ for the case $a_{0}=0.86$, resulting in very efficient transfer of energy from the wave to the particles. As the laser intensity increases, we find a value $p_{\mathrm{MAX}, x} \simeq 2 p_{\text {osc }, s w, x}$, slightly smaller than in the previous case. This is consistent with the fact that we infer an "optimal" damping of the SPW at $I \lambda_{0}^{2} \sim 10^{18} \mathrm{~W} \mathrm{~cm}^{-2} \mu \mathrm{m}^{2}$ where $\eta$ reaches a minimum, but absorption is large. Increasing the laser intensity above $I \lambda_{0}^{2} \sim 10^{18} \mathrm{~W} \mathrm{~cm}^{-2} \mu \mathrm{m}^{2}$, electron heating becomes somewhat less efficient, letting the wave grows to larger values.

Finally, we also performed simulations with fixed ions that show similar values of $\eta$ for the SPW if compared with the analogous mobile ion simulations. Thus, ion expansion does not affect the growth of the SPW for the short pulse interaction considered here.

\section{EFFECT OF DENSITY VARIATION}

In the following, we have varied the electron density from 25 to $100 n_{c}$ keeping the matching conditions for resonant excitation of the SPW. It should be noted that, as far as $n_{e} \gg n_{c}$, increasing $n_{e}$ further does not change drastically these matching conditions. This is interesting from an experimental point of view as the electron plasma density is a parameter hard to control with precision from laser-solid interaction experiment. In Table I, we report the laser absorption and $\eta$ as a function of the laser intensity for the two electron densities considered: 25 and $100 n_{c}$. We can 
TABLE I. Absorption and SPW field amplification factor, $\eta$, as a function of the laser intensity and the electron plasma density.

\begin{tabular}{lrcc}
\hline \hline$I \lambda_{0}^{2}\left(\mathrm{~W} \mathrm{~cm}^{-2} \mu \mathrm{m}^{2}\right)$ & $n_{e} / n_{c}$ & $\eta$ & Absorption (\%) \\
\hline $10^{16}$ & 25 & 4.6 & 54 \\
$10^{16}$ & 100 & 4.9 & 44 \\
$10^{18}$ & 25 & 3.3 & 62 \\
$10^{18}$ & 100 & 3.5 & 59 \\
$10^{19}$ & 25 & 4.2 & 75 \\
$10^{19}$ & 100 & 4.8 & 64 \\
\hline \hline
\end{tabular}

note that increasing the electron density does not change significantly the efficiency of the laser-SPW coupling as the value of $\eta$ stays roughly constant and slightly higher at $100 n_{c}$. More important, even if we have a decrease of the absorption at $100 n_{c}$, the value is nevertheless higher than that obtained at lower density when no SPW is excited; increasing the electron density only slightly affects the absorption enhancement. This behavior can be related to the decrease of the skin depth increasing the electron density up to $100 n_{c}$. However, especially at the highest intensities, the laser absorption is still very large, consistent with the idea that for $a_{0} \gtrsim 1$ the "enhanced vacuum heating" mechanism is important, and laser plasma coupling happens more efficiently than in the case of a flat interface. Hence, the SPW excitation may sustain large values of absorption also in high density regimes where basic collisionless mechanisms become less efficient.

\section{QUASI-STATIC MAGNETIC FIELD}

A strong quasi-static magnetic field is generated at the plasma surface when the SPW is excited. We will now analyze for $n_{e}=25 n_{c}$ this field $B_{z}$ (which is in the following normalized to $B_{0}=m_{e} c \omega / e \sim 107 \mathrm{MG}$ for $\lambda_{0}=1 \mu \mathrm{m}$ ) which was obtained averaging over one laser cycle.

Figure 4(a) shows the static magnetic field generated at the plasma surface for the pulse having $I \lambda_{0}^{2}$ $=10^{16} \mathrm{~W} \mathrm{~cm}^{-2} \mu \mathrm{m}^{2}$ at times $\omega t_{\text {Emax }}=460$ (as in Fig. 1 at the time at which the SPW field is maximum on the modulated surface). We observe the quasi-static magnetic field to be localized at the tips of the modulation as at this moment of the interaction, the electron density extends over a distance about $\sim k_{0}^{-1}$ as seen in Figure 4(b). The maximum positive value of the field is $\sim 0.023 m_{e} c \omega / e$ (2.5 MG for $\lambda_{0}=1 \mu \mathrm{m}$ ), while in the upper $y$ side of the dips the field is negative and reach $\sim 0.034 m_{e} c \omega / e$ (3.6 MG for $\lambda_{0}=1 \mu \mathrm{m}$ ). Later on the spatial distribution of the field evolves, the positive part tends to disappear. At a time when the pulse is over, for example, at $\omega t=600$ as reported in Figure 4(c), the field has negative values at any position, preserving its intensity compared to the time $\omega t_{\text {Emax }}=460$.

Figure 5 shows a plot of the maximum negative value of the magnetic field, generated by SPW excitation at $\omega t=600$, both as a function of (a) the maximum SPW field $E_{s w}$ (in order to compare with the theoretical prediction of Ref. 35) and (b) of the dimensionless amplitude $a_{0}$ of the laser pulse (which might be a more suitable parameter in experiments).
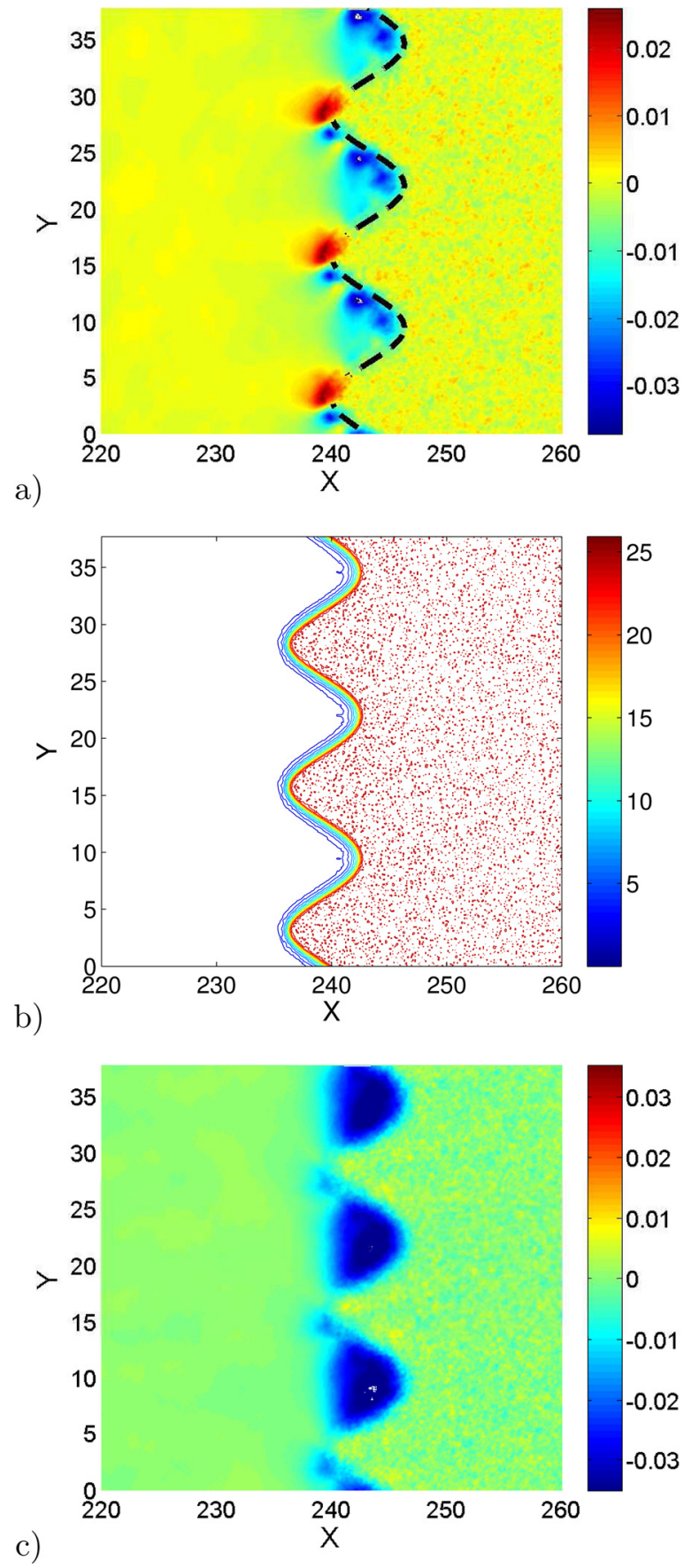

FIG. 4. (Color online) For $I \lambda_{0}^{2}=10^{16} \mathrm{~W} \mathrm{~cm}^{-2} \mu \mathrm{m}^{2}$, quasi static magnetic field in the $x y$ plane, at $\omega t_{E \max }=460$ (a) (in dotted line, the initial plasma surface is reported), and corresponding electron density isocontours from 1 to $25 n_{c}$ (b). Quasi static magnetic field in the $x y$ plane at a latter time $\omega t=600$ (c). The magnetic field is in units of $B_{0}$, and the lengths are in units of $k^{-1}$.

The scaling of the magnetic field with $E_{s w}$ is faster than linear for low intensities $\left(I \lambda_{0}^{2}<5 \times 10^{16} \mathrm{~W} \mathrm{~cm}^{-2} \mu \mathrm{m}^{2}\right.$, corresponding to the small frame of the figure) but almost linear for higher intensities, similar to the scaling with $a_{0}$.

A quasi-static magnetic field due to surface waves is predicted by an analytic hydrodynamic description of the SPW. ${ }^{35}$ Within this model, valid in the low field intensity range where $v_{t h}<v_{\text {osc }}$ and describing a flat surface, we find that the SPW field can induce (to the second order in the 

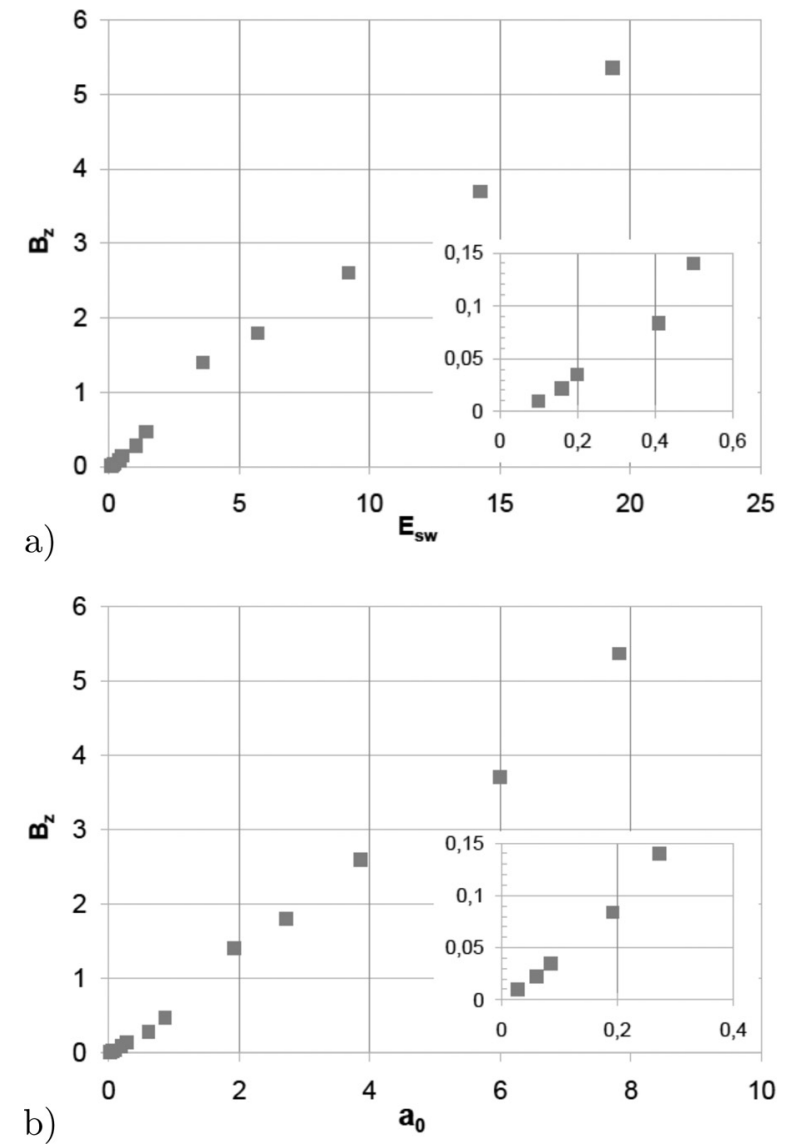

FIG. 5. Plot of the absolute value of the negative quasi-static magnetic field $\left|B_{z}\right|$ at $\omega t=600$ (a) as a function of the maximum of the SPW field $E_{s w}$ along the $x$-direction and (b) as a function of the $a_{0}$ value of the laser pulse which excites the SPW. The electric field is in units of $E_{0}$ and the magnetic field in units of $B_{0}$. In the small frame: zoom on the low intensity range.

amplitude of the SPW field) a static magnetic field in the skin depth of the overdense plasma. For an overdense cold plasma, the predicted normalised magnetic field is given by $B_{z}=2 \operatorname{sign}\{k\} \sqrt{n_{c} / n_{e}} E_{s w}^{2}$ (where $E_{s w}$ is the normalised electric field in units of $m_{e} c \omega / e$ ) and should be negative in the geometry used in our simulations. If we apply this formula to the simulations performed at $I \lambda_{0}^{2}=10^{15-16} \mathrm{~W} \mathrm{~cm}^{-2} \mu \mathrm{m}^{2}$, we find that the predicted field has the right order of magnitude about $\sim 1 \mathrm{MG}$ for $\lambda_{0}=1 \mu \mathrm{m}$, but it is systematically smaller than what found in the simulations. This can be related to the limit of validity of the hydrodynamic description used in Ref. 35 (no kinetic effects were taken into account, and a flat and sharp plasma-vacuum interface without grating was assumed). In order to check the possible effect of the density gradient at the surface, simulations with fixed ions at low intensity $I \lambda_{0}^{2}=10^{15-16} \mathrm{~W} \mathrm{~cm}^{-2} \mu \mathrm{m}^{2}$ have hence been performed in order to limit the expansion of the plasma towards vacuum and the presence of electrons outside the skin depth. In Figure 6, we show the magnetic field for the case $I \lambda_{0}^{2}=10^{16} \mathrm{~W} \mathrm{~cm}^{-2} \mu \mathrm{m}^{2}$ with immobile ions. In this case, there is a weak modifications of the SPW field that takes the value $E_{s w}=0.23$ instead of 0.2 with mobile ions (at $\omega t_{\text {Emax }}=460$ ). We can observe that the negative component of the magnetic field is localized in the plasma skin depth, and the absolute value of the field is reduced by a factor of 2 ,

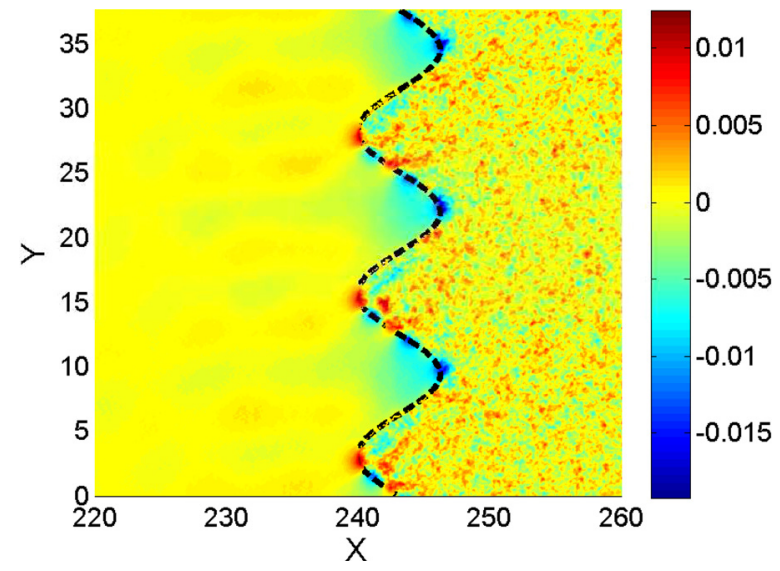

FIG. 6. (Color online) For $I \lambda_{0}^{2}=10^{16} \mathrm{~W} \mathrm{~cm}^{-2} \mu \mathrm{m}^{2}$ and in the case where the ions are fixed, quasi static magnetic field in the $x y$ plane, at $\omega t_{\text {Emax }}=460$. The magnetic field is given in units of $B_{0}$, and the lengths are in units of $k^{-1}$.

giving better agreement with the model prediction $B_{z} \sim-0.02$ from the formula above. However, at $I \lambda_{0}^{2}$ $=10^{15} \mathrm{~W} \mathrm{~cm}^{-2} \mu \mathrm{m}^{2}$, the variation in field values from the mobile to immobile ions case is only $10 \%$, and the predicted field is too small, so that we conclude that even at this low intensities kinetic effects are already important.

Analysis of the observed structure of the cycle-averaged magnetic field at late times (Fig. 4(c)) implies that, on the average, a positive current flows along the parallel $(y)$ direction on the vacuum side, while a negative return current flows in a thin surface layer. The positive current corresponds to a flow of electrons in the negative $y$ direction, consistently with the conservation of the laser wave momentum, since $k_{y}<0$. Since most of the momentum is absorbed by the suprathermal electrons, we conclude that those electrons produce the positive current from the average of their orbits on the vacuum side, i.e., in the course of a two-dimensional "vacuum heating" process. The return current is localized in the skin layer and it is due to slower bulk electrons with higher density. The structure of the steady magnetic field and of the associated currents is particularly clear after the end of the laser pulse in the case of low intensity since the flowing of the current on the modulated surface produces peak of the magnetic fields inside the dips of the surface modulation. It is also observed there that a low-density, quasi-neutral shelf is formed (we recall that the ions are mobile in this simulation) so that space-charge effects are quenched.

A similar behaviour is observed in the high intensity regime $\left(I \lambda_{0}^{2}>10^{18} \mathrm{~W} \mathrm{~cm}^{-2} \mu \mathrm{m}^{2}\right)$ where, however, the static magnetic field at later time shows a more complex structure; kinetic effects due to the electrons accelerated in the strong SPW field (the mentioned "enhanced vacuum heating" and $\vec{J} \times \vec{B}$ heating) are dominant and the emission of particles is very important, as shown by Fig. 7(b), where the density of electrons is shown at $\omega t \sim \omega t_{\text {Emax }}$, at $I \lambda_{0}^{2}=10^{19} \mathrm{~W} \mathrm{~cm}^{-2} \mu \mathrm{m}^{2}$. The streams of electrons in the vacuum strongly affect the magnetic field distribution, which is characterized by irregular negative and positive areas, as it can be seen in Fig. 7(a). As shown in Figure 7(c), this field evolves into a more regular one, but not as neat as in the low intensity case. Nevertheless, in all cases, the intensity of the magnetic field is increased by 


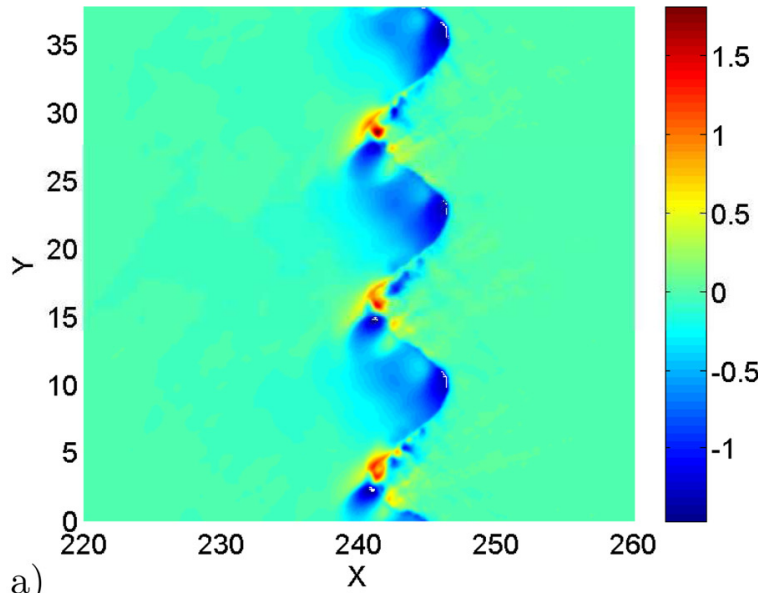

a)
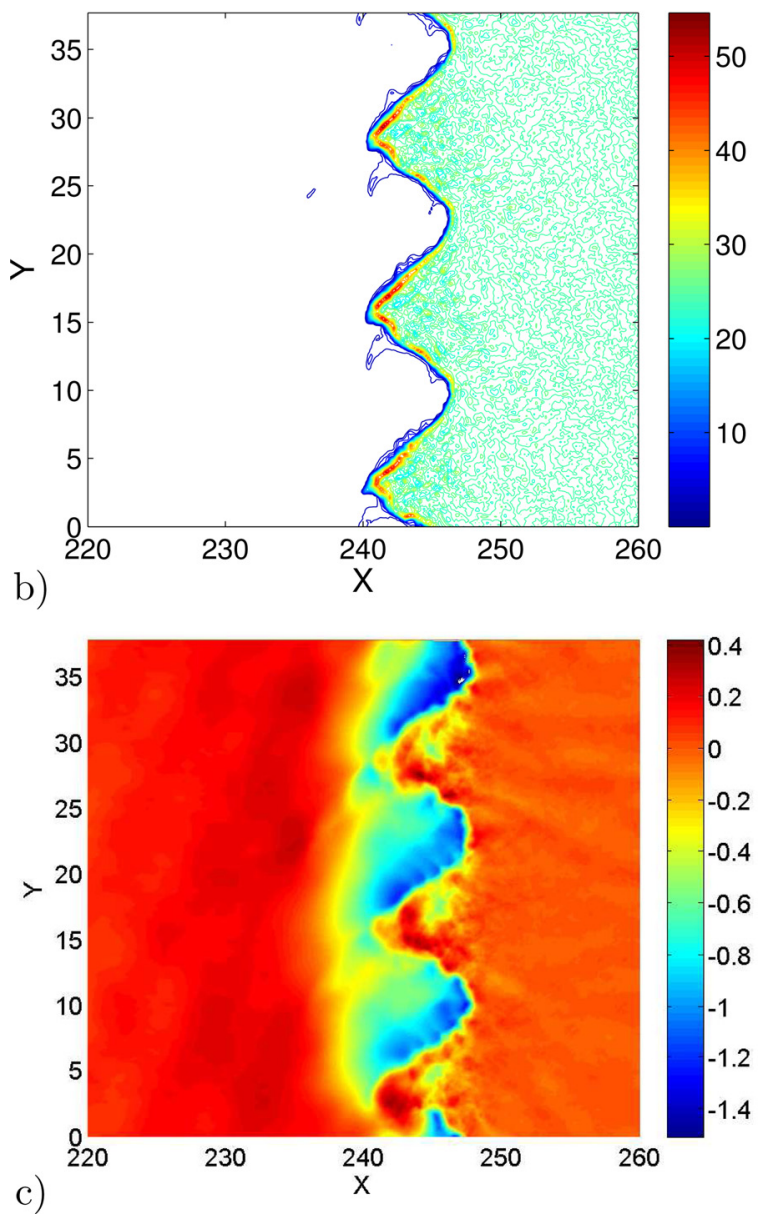

FIG. 7. (Color online) Quasi static magnetic field in the $x y$ plane, at $\omega t \sim \omega t_{E \max }=460$ (a) and corresponding electron density isocontours in the $x y$ plane (b) (given in particles per point). (c) Quasi static magnetic field in the $x y$ plane at $\omega t=600$. The laser intensity and the target density were $I \lambda_{0}^{2}=10^{19} \mathrm{~W} \mathrm{~cm}^{-2} \mu \mathrm{m}^{2}$ and $n_{e}=25 n_{c}$. The magnetic field in units of $B_{0}$, and the lengths are in units of $k^{-1}$.

SPW excitation even if, at relativistic intensities, the scaling with $E_{s w}$ becomes weaker. A net increase of the magnetic field is observed at the resonant surface where the maximum value reaches for $\lambda_{0}=1 \mu m \sim 190 \mathrm{MG}$ at $\omega t \sim \omega t_{\text {Emax }}$, while it is just $\sim 90 \mathrm{MG}$ for the flat interface. In the higher density case $n_{e}=100 n_{c}$, the magnetic field reaches values of $\sim 210 \mathrm{MG}$ for $\lambda_{0}=1 \mu \mathrm{m}$ at $I \lambda_{0}^{2}=\times 10^{19} \mathrm{~W} \mathrm{~cm}^{-2} \mu \mathrm{m}^{2}$.

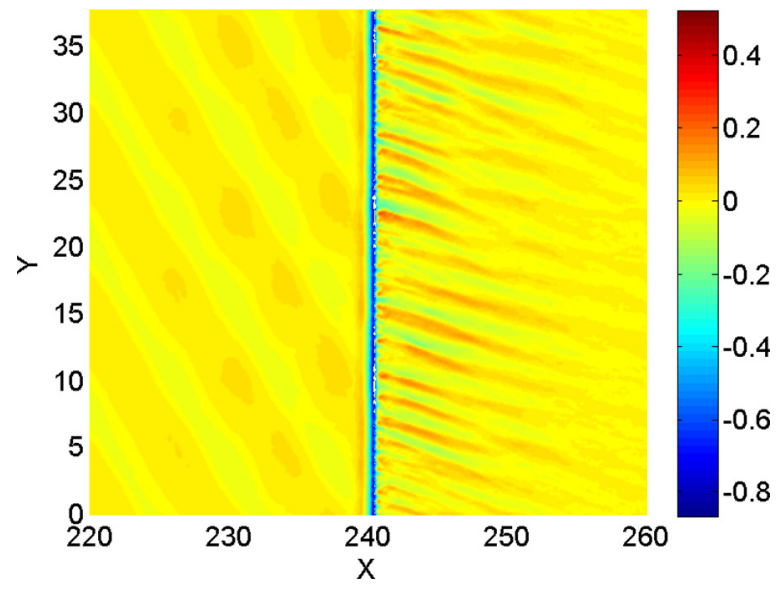

FIG. 8. (Color online) Quasi static magnetic field in the $x y$ plane, at $\omega t \sim \omega t_{\text {Emax }}=460$ in the case of a flat surface localized at $k x=240$ (no SPW). The laser intensity and the target density were $I \lambda_{0}^{2}=10^{19} \mathrm{~W} \mathrm{~cm}^{-2} \mu \mathrm{m}^{2}$ and $n_{e}=25 n_{c}$. The magnetic field in units of $B_{0}$, and the lengths are in units of $k^{-1}$.

Even if an exact comparison is not possible due to the difference in some parameters, we get in simulations where the SPW is not excited (case of the flat interface) values for the magnetic field, which are of the same order of what is found experimentally in Ref. 36 for the interaction of ultraintense laser pulses with a solid target and in previous simulations. ${ }^{24,37,38}$ A peculiar feature of flat interface simulations is the appearance of filamented structures in the magnetic fields as can be seen in Fig. 8, where the typical size of the filaments is $\sim \lambda_{0} / 6$, similar in size and in the time evolution to filaments observed and discussed by other authors. ${ }^{24,38}$ It is interesting to notice that a fundamental difference in the case when the SPW is excited is the absence of such filaments.

With SPW excitation, the maximum value obtained for the magnetic field is $\sim 580 \mathrm{MG}$ for $\lambda_{0}=1 \mu \mathrm{m}$ at $7 \times 10^{19} \mathrm{~W} \mathrm{~cm}^{-2} \mu \mathrm{m}^{2}$. Comparing with previous studies, different mechanisms for steady magnetic field generation in laser over-dense plasma interaction have been proposed which are of thermoelectric origin and involved gradient electron density or temperature anisotropy. ${ }^{26,39,40}$ We find here that the magnetic field observed when SPW is excited is much larger than what found when no plasma wave is excited. We can thus look at the particle motion in the static field. In the relativistic regime, the Larmor radius, $R_{L}$, equals $\lambda_{0} \gamma v /\left(B_{z} 2 \pi c\right)$, where $B_{z}$ is the normalised magnetic field and $v$ the electron velocity. If we calculate the ratio $R_{L} / \lambda_{0}$ by taking the values of $B_{z}, \gamma \sim \gamma_{o s c, s w}$, and $v \sim v_{\text {osc,sw }}(\sim c)$ found in the simulations, we have that for the most energetic particles in the relativistic regime, $R_{L} / \lambda_{0} \sim 1$. Since the typical size of the layer where the static magnetic field is present is of the order $\lambda_{0}$, this implies that the magnetic field may confine particles near the surface, a point that may be important for potential applications.

\section{CONCLUSION}

The efficiency of laser overdense plasma coupling via surface plasma wave excitation has been investigated over a wide range of pulse intensity from $10^{15}$ to $10^{20} \mathrm{~W} \mathrm{~cm}^{-2} \mu \mathrm{m}^{2}$ 
at $25-100 n_{c}$ with $2 D$ relativistic PIC simulations where a plasma wave was resonantly excited at the surface of a modulated plasma target. We find for all the range of laser intensities considered a strong efficiency of the laser plasma coupling; absorption is significantly greater than in the case of a flat surface for the same parameters, reaching at $a_{0}=6$ $\left(I \lambda_{0}^{2}=5 \times 10^{19} \mathrm{~W} \mathrm{~cm}^{-2} \mu \mathrm{m}^{2}\right)$ values up to $75 \%$ for $n_{e}=25 n_{c}$ and up to $64 \%$ for $n_{e}=100 n_{c}$, respectively, also showing only a weak decrease for increasing plasma density. The excitation of the surface wave also leads to an increase in amplitude and spatial extension of the steady magnetic field near the laser-plasma interface. The self-generated magnetic field is strong enough to confine "fast" laseraccelerated electrons.

For relatively low, sub-relativistic irradiance $\left(I \lambda_{0}^{2} \lesssim 10^{18} \mathrm{~W} \mathrm{~cm}^{-2} \mu \mathrm{m}^{2}\right.$ or $\left.a_{0} \lesssim 1\right)$, the SPW amplification factor attains the highest values but decreases with increasing $a_{0}$. The static magnetic field has a faster than linear scaling with the laser intensity and may reach values of a few megagauss. For higher, relativistic irradiance $I \lambda_{0}^{2} \gtrsim 10^{18} \mathrm{~W} \mathrm{~cm}^{-2} \mu \mathrm{m}^{2}$ or $a_{0} \gtrsim 1$, both the amplification factor and the fractional absorption grow slowly with laser intensity. They saturate for $a_{0} \gtrsim 4$, while the steady magnetic field scales almost linearly with $a_{0}$ and may reach values exceeding of several hundreds of megagauss. These different scalings suggest that kinetic and relativistic effects become important at high intensity $a_{0}>1$. These results show that resonant surface wave excitation leads to efficient laser-plasma coupling and high efficiency also at strongly relativistic intensities. The steady magnetic field may influence the generation and transport of fast electrons inside the target bulk, a point that will be the topic of further investigation.

\section{ACKNOWLEDGMENTS}

This work was sponsored by CNR (Italy) and CNRS (France) through the CNR-CNRS agreement for scientific cooperation (SWILAP project). Usage of the CEA computing center CCRT under the Project No. 559 is also thankfully acknowledged.

${ }^{1}$ G. A. Mourou, T. Tajima, and S. V. Bulanov, Rev. Mod. Phys. 78, 309 (2006).

${ }^{2}$ C. Thaury, F. Qur, J.-P. Geindre, A. Levy, T. Ceccotti, P. Monot, M. Bougeard, F. Rau, P. dOliveira, P. Audebert, R. Marjoribanks and Ph. Martin, Nat. Phys. 3, 424 (2007).

${ }^{3}$ S. C. Wilks and W. L. Kruer, IEEE J. Quantum Electron. 33, 1954 (1997).

${ }^{4}$ P. J. Catto and M. R. More, Phys. Fluids 20, 704 (1997).

${ }^{5}$ W. L. Kruer and K. Estabrook, Phys. Fluids 28, 430 (1985).

${ }^{6}$ F. Brunel, Phys. Rev. Lett. 59, 52 (1987); Phys. Fluids 31, 2714 (1988).

${ }^{7}$ E. G. Gamaliy and R. Dragila, Phys. Rev. A 42, 929 (1990).

${ }^{8}$ C. Ren, M. Tzoufras, J. Tonge, W. B. Mori, F. S. Tsung, M. Fiore, R. A. Fonseca, L. O. Silva, J. C. Adam, and A. Heron, Phys. Plasmas 13, 056308 (2006).

${ }^{9}$ R. N. Sudan, Phys. Rev. Lett. 70, 3075 (1993).
${ }^{10}$ R. J. Mason and M. Tabak, Phys. Rev. Lett. 80, 524 (1998).

${ }^{11}$ J. C. Gauthier, S. Bastiani, P. Audebert, J. P. Geindre, K. Neuman, T. D. Donnelly, R. W. Falcone, R. L. Shepherd, D. F. Price, and W. E. White, Proc. SPIE 2523, 242 (1995).

${ }^{12}$ J. Kupersztych, M. Raynaud, and C. Riconda, Phys. Plasmas 11, 1669 (2004).

${ }^{13}$ M. Raynaud, J. Kupersztych, C. Riconda, J. C. Adam, and A. Heron, Phys. Plasmas 14, 092702 (2007).

${ }^{14}$ G. Hu, A. Lei, J. Wang, L. Huang, W. Wang, X. Wang, Y. Xu, B. Shen, J. Liu, W. Yu, R. Li, and Z. Xu, Phys. Plasmas 17, 083102 (2010).

${ }^{15}$ X. Lavocat-Dubuis and J.-P. Matte, Phys. Plasmas 17, 093105 (2010); Y. T. Li, X. H. Yuan, M. H. Xu, Z. Y. Zheng, Z. M. Sheng, M. Chen, Y. Y. Ma, W. X. Liang, Q. Z. Yu, Y. Zhang, F. Liu, Z. H. Wang, Z. Y. Wei, W. Zhao, Z. Jin, and J. Zhang, Phys. Rev. Lett. 96, 165003 (2006); W.-M. Wang, Z.-M. Sheng, and J. Zhang, Phys. Plasmas 15, 030702 (2008); G. Hu, A. Lei, W. Wang, X. Wang, L. Huang, J. Wang, Y. Xu, J. Liu, W. Yu, B. Shen, R. Li, and Z. Xu, Phys. Plasmas 17, 033109 (2010).

${ }^{16}$ O. Klimo, J. P. Psikal, J. Limpouch, J. Proska, F. Novotny, T. Ceccotti, V. Floquet, and S. Kawata, New J. Phys. 13, 053028 (2011).

${ }^{17}$ P. K. Kaw and J. B. McBride, Phys. Fluids 13, 1784 (1970).

${ }^{18}$ H. Chen, S. C. Wilks, D. D. Meyerhofer, J. Bonlie, C. D. Chen, S. N. Chen, C. Courtois, L. Elberson, G. Gregori, W. Kruer, O. Landoas, J. Mithen, J. Myatt, C. D. Murphy, P. Nilson, D. Price, M. Schneider, R. Shepherd, C. Stoeckl, M. Tabak, R. Tommasini and P. Beiersdorfer, Phys. Rev. Lett. 105, 015003 (2010).

${ }^{19}$ R. W. Lee, S. J. Moon, H. K. Chung, W. Rosmus, H. A. Baldis, G. Gregori, R. C. Cauble, O. L. Landen, J. S. Wark, A. Ng, S. J. Rose, C. L. Lewis, D. Riley, J. C. Gauthier and P. Auderbert, J. Opt. Soc. Am. B 20, 770 (2003).

${ }^{20}$ P. McKenna, A. P. L. Robinson, D. Neely, M. P. Desjarlais, D. C. Carroll, M. N. Quinn, X. H. Yuan, C. M. Brenner, M. Burga, M. Coury, P. Gallegos, R. J. Gray, K. L. Lancaster, Y. T. Li, X. X. Lin, O. Tresca and C. G. Wahlstrom, Phys. Rev. Lett. 106, 185004 (2011).

${ }^{21}$ V. S. Belyaev, V. P. Krainov, V. S. Lisitsa, and A. P. Matafonov, Physics (Uspekhi) 51, 793 (2008); and references herein.

${ }^{22}$ J. A. Stamper, K. Papadopoulos, R. N. Sudan, S. O. Dean, E. A. McLean, and J. M. Dawson, Phys. Rev. Lett. 26, 1012 (1971).

${ }^{23}$ A. Pukhov and J. Meyer-ter-Vehn, Phys. Rev. Lett. 76, 3975 (1996).

${ }^{24}$ J. C. Adam, A. Heron, and G. Laval, Phys. Rev. Lett. 97, 205006 (2006).

${ }^{25}$ H. Cai, S. Zhu, M. Chen, S. Wu, X. T. He, and K. Mima, Phys. Rev. E 83, 036408 (2011).

${ }^{26}$ R. J. Mason, E. S. Dodd, and B. J. Albright, Phys. Rev. E 72, 015401(R) (2005)

${ }^{27}$ J. M. Kindel, K. Lee, and E. L. Lindman, Phys. Rev. Lett. 34, 134 (1975).

${ }^{28}$ A. Macchi, F. Cornolti, F. Pegoraro, T. V. Liseikina, H. Ruhl, and V. A. Vshivkov, Phys. Rev. Lett. 87, 205004 (2001).

${ }^{29}$ Developed at CPhT, Ecole Polytechnique.

${ }^{30}$ P. Gibbon and A. Bell, Phys. Rev. Lett. 68, 1535 (1992).

${ }^{31}$ P. Mulser and D. Bauer, High Power Laser-Matter Interaction (Springer, New York, 2010).

${ }^{32}$ Y. Ping, R. Shepherd, B. F. Lasinski, M. Tabak, H. Chen, H. K. Chung, K. B. Fournier, S. B. Hansen, A. Kemp, D. A. Liedahl, K. Widmann, S. C. Wilks, W. Rozmus and M. Sherlock, Phys. Rev. Lett. 100, 085004 (2008).

${ }^{33}$ J. P. Geindre, R. S. Marjoribanks, and P. Audebert, Phys. Rev. Lett. 104, 135001 (2010).

${ }^{34}$ P. Mulser, D. Bauer, and H. Ruhl, Phys. Rev. Lett. 101, 225002 (2008).

${ }^{35}$ A. Bigongiari, M. Raynaud, and C. Riconda, Phys. Rev. E 84, 015402(R) (2011).

${ }^{36}$ A. Gopal, M. Tatarakis, F. N. Beg, E. L. Clark, A. E. Dangor, R. G. Evans, P. A. Norreys, M. S. Wei, M. Zepf, and K. Krushelnick, Phys. Plasmas 15, 122701 (2008).

${ }^{37}$ S. C. Wilks, W. L. Kruer, M. Tabak, and A. B. Langdon, Phys. Rev. Lett. 69, 1383 (1992).

${ }^{38}$ Y. Sentoku, K. Mima, S. Kojima, and H. Ruhl, Phys. Plasmas 7, 689 (2000).

${ }^{39}$ D. W. Forslund and J. U. Brackbill, Phys. Rev. Lett. 48, 1614 (1982).

${ }^{40}$ C. Thaury, P. Mora, A. Heron, and J. C. Adam, Phys. Rev. E 82, 016408 (2010). 